\title{
Generalization of radiative jet energy loss to non-zero magnetic mass
}

\author{
Magdalena Djordjevic ${ }^{1}$ and Marko Djordjevic ${ }^{2}$ \\ ${ }^{1}$ Institute of Physics Belgrade, University of Belgrade, Serbia \\ ${ }^{2}$ Faculty of Biology, University of Belgrade, Serbia
}

(Dated: October 29, 2018)

\begin{abstract}
Reliable predictions for jet quenching in ultra-relativistic heavy ion collisions require accurate computation of radiative energy loss. With this goal, an energy loss formalism in a realistic finite size dynamical QCD medium was recently developed. While this formalism assumes zero magnetic mass - in accordance with the one-loop perturbative calculations - different non-perturbative approaches report a non-zero magnetic mass at RHIC and LHC. We here generalize the energy loss to consistently include a possibility for existence of non-zero magnetic screening. We also present how the inclusion of finite magnetic mass changes the energy loss results. Our analysis indicates a fundamental constraint on magnetic to electric mass ratio.
\end{abstract}

PACS numbers: 25.75.-q, 25.75.Nq, 12.38.Mh, 12.38.Qk

\section{INTRODUCTION}

Heavy flavor suppression is considered to be a powerful tool to study the properties of a QCD medium created in ultra-relativistic heavy ion collisions [1]. The suppression results from the energy loss of high energy partons moving through the plasma [2]. Therefore, reliable computations of heavy quark energy loss are essential for the reliable predictions of jet suppression. In [3, 4], we developed a theoretical formalism for the calculation of the first order in opacity radiative energy loss in a dynamical QCD medium (see also a viewpoint in [5]). That study models radiative energy loss in a realistic finite size QCD medium with dynamical constituents, therefore removing a major approximation of static scattering centers present in previous calculations (see e.g. 6 - 12]).

The dynamical energy loss formalism [3, 4] is based on HTL perturbative QCD, which requires zero magnetic mass. However, different non-perturbative approaches [13 16] suggest a non-zero magnetic mass at RHIC and LHC. This, therefore, arises a question if finite magnetic mass can be consistently included in the dynamical energy loss calculations, and how this inclusion would modify the energy loss results.

\section{RADIATIVE ENERGY LOSS IN A DYNAMICAL QCD MEDIUM}

In [3, 4], we used finite temperature field theory (HTL approximation) and calculated the radiative energy loss in a finite size dynamical QCD medium. The obtained expression for the energy loss is given by Eq. (1):

$$
\frac{\Delta E_{\mathrm{rad}}}{E}=\frac{C_{R} \alpha_{s}}{\pi} \frac{L}{\lambda_{\mathrm{dyn}}} \int d x \frac{d^{2} k}{\pi} \frac{d^{2} q}{\pi} v(\boldsymbol{q}) f(\boldsymbol{k}, \boldsymbol{q}, x),
$$

where

$$
\begin{aligned}
f(\boldsymbol{k}, \boldsymbol{q}, x) & =2\left(1-\frac{\sin \frac{(\boldsymbol{k}+\boldsymbol{q})^{2}+\chi}{x E^{+}} L}{\frac{(\boldsymbol{k}+\boldsymbol{q})^{2}+\chi}{x E^{+}} L}\right) \\
& \times \frac{(\boldsymbol{k}+\boldsymbol{q})}{(\boldsymbol{k}+\boldsymbol{q})^{2}+\chi}\left(\frac{(\boldsymbol{k}+\boldsymbol{q})}{(\boldsymbol{k}+\boldsymbol{q})^{2}+\chi}-\frac{\boldsymbol{k}}{\boldsymbol{k}^{2}+\chi}\right)
\end{aligned}
$$

In Eqs. (11) and (2), $L$ is the length of the finite size dynamical QCD medium and $E$ is the jet energy. $\boldsymbol{k}$ is transverse momentum of radiated gluon, while $\boldsymbol{q}$ is transverse momentum of the exchanged (virtual) gluon. $\alpha_{s}=\frac{g^{2}}{4 \pi}$ is coupling constant and $C_{R}=\frac{4}{3} . \quad v(\boldsymbol{q})$ is the effective crossection in dynamical QCD medium and $\lambda_{\text {dyn }}^{-1} \equiv C_{2}(G) \alpha_{s} T=3 \alpha_{s} T\left(C_{2}(G)=3\right)$ is defined as "dynamical mean free path" (see also [17]). $\chi \equiv M^{2} x^{2}+m_{g}^{2}$, where $x$ is the longitudinal momentum fraction of the heavy quark carried away by the emitted gluon, $M$ is the mass of the heavy quark, $m_{g}=\mu_{E} / \sqrt{2}$ is the effective mass for gluons with hard momenta $k>T$ [18], and $\mu_{E}$ is the Debye mass. We assume constant coupling $g$. Furthermore, we note that in Eq. (11) effective crossection $v(\boldsymbol{q})$ represents the interaction between the jet and exchanged gluon, while $f(\boldsymbol{k}, \boldsymbol{q}, x)$ represents the interaction between the jet and radiated gluon [3, 4].

The goal of this section is to start from the above expression, and generalize it to include the existence of nonzero magnetic mass 13 16. To proceed, we note that the inclusion of magnetic mass modifies the gluon self energy, and therefore our goal is to study how modified self energy of radiated and exchanged gluons change the energy loss result. We also note that from [3], it is straightforward to show that non-zero magnetic mass does not alter the factorization $(v(\boldsymbol{q}) f(\boldsymbol{k}, \boldsymbol{q}, x))$ in the integrand of Eq. (1), due to the fact that the factorization does not depend on specific form of self energy. Since $v(\boldsymbol{q})$ depends only on the exchanged gluon self energy, while $f(\boldsymbol{k}, \boldsymbol{q}, x)$ depends only on a radiative gluon self energy, we below separately study how the inclusion of magnetic mass will modify $v(\boldsymbol{q})$ and $f(\boldsymbol{k}, \boldsymbol{q}, x)$. 
Modification of the effective crossection due to magnetic screening. The effective crossection $v(\boldsymbol{q})$ can be written in the following form

$$
v(\boldsymbol{q})=v_{L}(\boldsymbol{q})-v_{T}(\boldsymbol{q}),
$$

where $v_{T}(\boldsymbol{q})\left(v_{L}(\boldsymbol{q})\right)$ is transverse (longitudinal) contribution to the effective crossection, given by [3, 19]

$$
v_{T, L}(\boldsymbol{q})=\frac{1}{\boldsymbol{q}^{2}+\operatorname{Re} \Pi_{T, L}(\infty)}-\frac{1}{\boldsymbol{q}^{2}+\operatorname{Re} \Pi_{T, L}(0)},
$$

where $\Pi_{T}$ and $\Pi_{L}$ are gluon self energies. While in [3, 44] the derivation of the effective crossection was made through a hard thermal loop for the self-energy $\Pi$, one should note that the crossection does not depend on specific form of gluon self energy [19]. That is, the expression is valid for any self-energy satisfying the following assumptions [19]:

1. $\Pi$ depends only on $x \equiv k_{0} / k$

2. $\operatorname{Im} \Pi(x=0)=0$

3. $\operatorname{Im} \Pi(x)=0$ if $x \geq 1$

4. $\operatorname{Re} \Pi(x) \geq 0$ if $x \geq 1$,

which are reasonable approximations for any system of well defined quasiparticles.

Therefore, we see that the result given by Eq. (4) depends only on four numbers: $\operatorname{Re} \Pi_{T, L}(\infty)$ and $\operatorname{Re} \Pi_{T, L}(0)$; due to this, we don't need to know the full gluon propagator to generalize the effective crossection to the case of finite magnetic screening. The first two numbers are the masses of the longitudinal and the transverse gluons at zero momentum (so called plasmon masses). These are shown to be equal due to Slavnor-Taylor identities 20 22]. Physically, this property means that there is no way to distinguish transverse and longitudinal modes for a particle at rest [19]. Therefore, we need only to introduce one plasmon mass:

$$
\operatorname{Re} \Pi_{T}(\infty)=\operatorname{Re} \Pi_{L}(\infty) \equiv \mu_{p l}^{2} .
$$

The second two quantities are squares of the screening masses for the transverse and longitudinal static gluon exchanges. The longitudinal (electric) screening mass is the familiar Debye mass:

$$
\mu_{E}^{2} \equiv \operatorname{Re} \Pi_{L}(0)
$$

In the HTL approximation, there is no screening for the transverse static gluons, but this is not expected to hold generally. The corresponding screening mass is the magnetic mass, and is denoted

$$
\mu_{M}^{2} \equiv \operatorname{Re} \Pi_{T}(0) .
$$

The general expressions for the transverse and longitudinal contributions to the effective crossections $v_{T, L}(\boldsymbol{q})$ then become

$$
v_{L, T}(\boldsymbol{q})=\frac{1}{\left(\boldsymbol{q}^{2}+\mu_{p l}^{2}\right)}-\frac{1}{\left(\boldsymbol{q}^{2}+\mu_{E, M}^{2}\right)}
$$

After replacing the expressions for $v_{L, T}(\boldsymbol{q})$ from Eq. (8) into Eq. (3), we finally obtain the expression for the effective crossection in the case of non-zero magnetic mass:

$$
v(\boldsymbol{q})=\frac{\mu_{E}^{2}-\mu_{M}^{2}}{\left(\boldsymbol{q}^{2}+\mu_{M}^{2}\right)\left(\boldsymbol{q}^{2}+\mu_{E}^{2}\right)} .
$$

Note that $v(\boldsymbol{q})$ in Eq. (9) does not depend on plasmon mass. In other words, all the dependence on the plasmon mass drops out in this expression. This seems reasonable given that $v(\boldsymbol{q})$ involves only space-like gluon exchanges (see [3, 4, 17]), while the plasmon mass is a property of time-like gluons [19]. Therefore, we only need to know the two screening masses $\mu_{E}$ and $\mu_{M}$, in order to generalize the effective crossection to non-zero magnetic mass.

Modification of $f(\boldsymbol{k}, \boldsymbol{q}, x)$ due to magnetic screening. As we discussed above, the introduction of the magnetic mass leads to the modification of the exchanged and radiated gluon self energy. In this subsection, we study how the introduction of the magnetic mass in the radiated gluon self energy modifies the radiative energy loss.

To proceed with this study, we note that all radiative energy loss calculations [3, 6 12, 23] are performed by assuming validity of the soft gluon $(\omega \ll E)$ and soft rescattering $\left(\omega \gg|\boldsymbol{k}| \sim|\boldsymbol{q}| \sim q_{0}, q_{z}\right.$ ) approximations. Within these approximations, we showed that in the finite temperature QCD medium radiated gluons have similar dispersion relation as in the vacuum, with the difference that the gluons now acquire a "mass" [18]. We also showed that the gluon mass in the medium is approximately equal to the value of gluon self energy at $x=1[18,24]$ (so called asymptotic mass $m_{\infty}=\sqrt{\Pi_{T}(x=1)}$ ).

Therefore, analogously to the previous section, we see that the dependence of the $f(\boldsymbol{k}, \boldsymbol{q}, x)$ on gluon self energy reduces to just a single number: $\Pi_{T}(x=1)$, which is defined as a square of gluon mass $m_{g}$. Due to this, instead of knowing the full gluon propagator, we only need to know how $m_{g}$ changes in order to obtain how $f(\boldsymbol{k}, \boldsymbol{q}, x)$ is modified in the case of non-zero magnetic mass.

In principle, gluon mass may change with the introduction of non-zero magnetic screening, but (to our knowledge) no study up to now addressed how nonperturbative calculations would modify the gluon asymptotic mass. Consequently, our approach in the next section is to introduce an ansatz in order to numerically investigate how perturbations of $m_{g}$, for a magnitude corresponding to magnetic mass, change radiative energy loss results. 
Modification of the energy loss expression due to magnetic screening. After replacing the effective crossection $v(\boldsymbol{q})$ (see Eq. (9)) into Eq. (1), the total energy loss becomes

$$
\begin{array}{r}
\frac{\Delta E_{\mathrm{rad}}}{E}=\frac{C_{R} \alpha_{s}}{\pi} \frac{L}{\lambda_{\mathrm{dyn}}}\left(\mu_{E}^{2}-\mu_{M}^{2}\right) \int d x \frac{d^{2} k}{\pi} \frac{d^{2} q}{\pi} \\
\times \frac{1}{\left(\boldsymbol{q}^{2}+\mu_{M}^{2}\right)\left(\boldsymbol{q}^{2}+\mu_{E}^{2}\right)} f(\boldsymbol{k}, \boldsymbol{q}, x),
\end{array}
$$

where $f(\boldsymbol{k}, \boldsymbol{q}, x)$ is given by Eq. (2). Note that in Eq. (2), $\chi \equiv M^{2} x^{2}+M_{g}^{2}$, where the gluon mass $M_{g}$ can now be different from $m_{g}=\mu_{E} / \sqrt{2}$ (see previous subsection).

A constraint on the magnetic mass range. We first discuss one interesting qualitative observation, that comes directly from Eq. (10): Since integrand in Eq. (10) is positive definite, if magnetic mass becomes larger than electric mass, the net energy loss becomes negative. Therefore, if magnetic mass is larger than electric mass, the quark jet would, overall, start to gain (instead of lose) energy in this type of plasma. The origin for this effect can be traced from Eq. (8): if the magnetic mass is larger than electric mass, the energy gain from magnetic contribution becomes so large, that it, overall, leads to the total energy gain of the jet. One should note that such a gain would involve transfer of energy of disordered motion of plasma constituents, to energy of ordered jet motion. Such transfer of "low" to "high" quality energy would be in a violation of the second law of thermodynamics. From this follows that it is impossible to create a plasma with magnetic mass larger than electric, which places a fundamental limit on magnetic mass range. Indeed, in an agreement with this limit, various non-perturbative approaches [13 16] suggest that, at RHIC and LHC, $0.4<\mu_{M} / \mu_{E}<0.6$.

\section{NUMERICAL RESULTS}

In this section, we numerically study how the inclusion of non-zero magnetic mass modifies the energy loss results. To address this, we consider a quark-gluon plasma of temperature $T=225 \mathrm{MeV}$, with $n_{f}=2.5$ effective light quark flavors and strong interaction strength $\alpha_{s}=0.3$, as representative of average conditions encountered in $\mathrm{Au}+\mathrm{Au}$ collisions at RHIC. For the light quark jets we assume that their mass is dominated by the thermal mass $M=\mu / \sqrt{6}$, where $\mu=g T \sqrt{1+N_{f} / 6} \approx 0.5$ $\mathrm{GeV}$ is the Debye screening mass. The charm mass is taken to be $M=1.2 \mathrm{GeV}$, and for the bottom mass we use $M=4.75 \mathrm{GeV}$. To simulate (average) conditions in $\mathrm{Pb}+\mathrm{Pb}$ collisions at the $\mathrm{LHC}$, we use the temperature of the medium of $T=400 \mathrm{MeV}$.

We first investigate how possible changes of gluon mass (i.e. $f(\boldsymbol{k}, \boldsymbol{q}, x))$ due to non-zero magnetic screening may change radiative energy loss (see the previous section). To investigate this, we introduce an ansatz that both electric and magnetic masses equally contribute to gluon self energy at $x=1$ (i.e. $M_{g}=\sqrt{\frac{\mu_{E}^{2}+\mu_{M}^{2}}{2}}$ ). With this ansatz, which changes the gluon mass for a magnitude comparable to magnetic mass, we obtain a negligible change in radiative energy loss (data not shown). For simplicity, we will therefore further assume that the gluon mass of radiated gluon remains the same as in 3,18 , i.e. that $M_{g}=\mu_{E} / \sqrt{2}$. Consequently, in the rest of this section, we numerically study how the inclusion of magnetic mass into the effective crossection modifies the energy loss results compared to the results presented in [3].

Energy loss dependence on the magnetic mass is shown in Fig. 1 for RHIC and LHC case. As expected, we see that energy loss decreases with the increase in magnetic mass. Note that, when magnetic masses becomes larger than electric mass, the net energy loss becomes negative, as discussed in the previous section. In Fig. 2, we show momentum dependence of fractional energy loss, where we concentrate on the range $0.4<\mu_{M} / \mu_{E}<0.6$, as suggested by various non-perturbative approaches 13 16]. We see that finite magnetic mass reduces the energy loss in dynamical QCD medium by $25 \%$ to $50 \%$.

We note that, contrary to what one may naively expect, majority of the energy loss decrease does not come from the introduction of magnetic screening in the denominator of the effective crossection (see Eq. (90)). In fact, the major decrease in the energy loss actually comes from the presence of the magnetic mass in the numerator of the energy loss expression (numerical results not shown). For example, for the ratio $\mu_{M} / \mu_{E}=0.5,25 \%$ decrease in the energy loss comes from the presence of the magnetic mass in the numerator, while only $14 \%$ decrease comes from the presence of magnetic screening in the denominator of the effective crossection. The reason behind this is that introduction of magnetic screening in the denominator of effective crossection does not regulate the logarithmic divergence, as might be expected from Eq. (10). This is because this divergence is already naturally regulated in Eq. (11), where all the relevant diagrams are taken into account $[3,4]$.

\section{SUMMARY}

This paper generalizes dynamical energy loss formalism to non-zero magnetic screening. While the introduction of magnetic mass into any perturbative calculation is inherently phenomenological, the presented inclusion of the effects of modified gluon self energy into our radiative energy loss formalism is of general validity as long as a well defined quasiparticle system is assumed. Analysis of the finite magnetic mass effects leads to a constraint that it is impossible to create a plasma with magnetic 

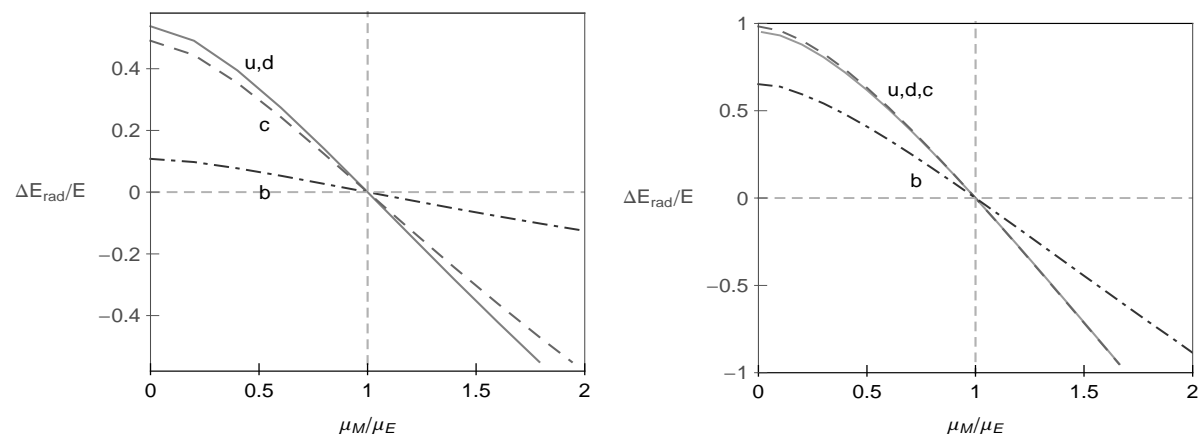

FIG. 1: Fractional radiative energy loss is shown as a function of magnetic and electric mass ratio. Assumed path length is $L=5 \mathrm{fm}$ and initial jet energy is 10 (50) GeV for a left (right) panel. Full, dashed and dot-dashed curves correspond to light, charm and bottom quark respectively. Note that for left (right) panel, we assume RHIC (LHC) conditions, with a medium of temperature $T=225$ (400) $\mathrm{MeV}$.
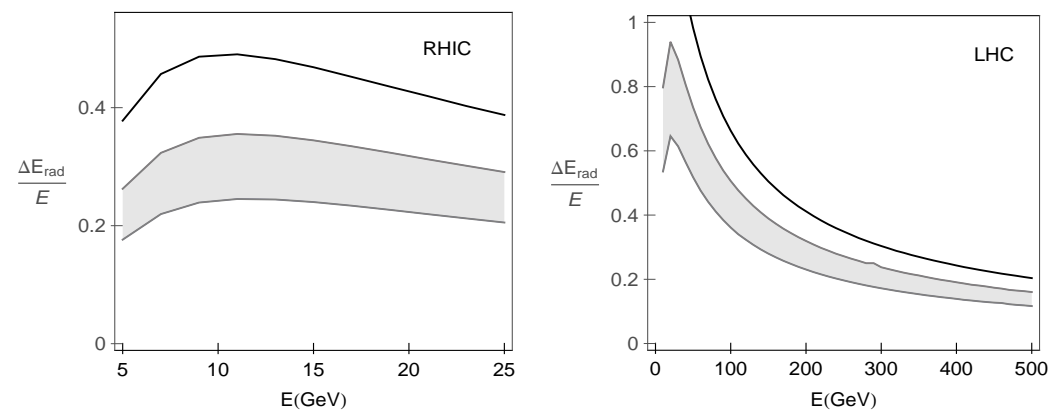

FIG. 2: Fractional radiative energy loss for an assumed path length $L=5 \mathrm{fm}$ as a function of momentum for charm quarks. Left (right) panel corresponds to RHIC (LHC) conditions. Full curve corresponds to the case when magnetic mass is zero. Gray band corresponds to the energy loss when magnetic mass is non-zero (i.e. $0.4<\mu_{M} / \mu_{E}<0.6$ ). Upper (lower) boundary of the band corresponds to the case $\mu_{M} / \mu_{E}=0.4$ $\left(\mu_{M} / \mu_{E}=0.6\right)$.

mass larger than electric. Results presented in this paper allow including non-zero magnetic screening into jet suppression calculations, and open a possibility for more accurate mapping of QGP properties.

Acknowledgments: Valuable discussions with Joseph Kapusta, Antony Rebhan, and Miklos Gyulassy are gratefully acknowledged. This work is supported by Marie Curie International Reintegration Grant within the $7^{\text {th }}$ European Community Framework Programme (PIRG08-GA-2010-276913) and by the Ministry of Science and Technological Development of the Republic of Serbia, under projects No. ON171004 and ON173052. Marko Djordjevic is supported in part by Marie Curie International Reintegration Grant within the $7^{\text {th }}$ European Community Framework Programme (PIRG08-GA2010-276996).

[1] N. Brambilla et al., Preprint hep-ph/0412158 (2004).

[2] M. Guylassy, I. Vitev, X. N. Wang and B. W. Zhang, in Quark Gluon Plasma 3, edited by R. C. Hwa and X. N. Wang, p. 123 (World Scientific, Singapore, 2003)

[3] M. Djordjevic, Phys. Rev. C 80, 064909 (2009).

[4] M. Djordjevic and U. Heinz, Phys. Rev. Lett. 101, 022302 (2008).

[5] M. Gyulassy, Physics 2, 107 (2009).

[6] M. Gyulassy, P. Levai and I. Vitev, Nucl. Phys. B 594, 371 (2001).

[7] M. Gyulassy and X. N. Wang, Nucl. Phys. B 420, 583 (1994); X. N. Wang, M. Gyulassy and M. Plumer, Phys. Rev. D 51 (1995) 3436.
[8] U.A. Wiedemann, Nucl. Phys. B 588, 303 (2000); and Nucl. Phys. B 582, 409 (2000).

[9] E. Wang and X. N. Wang, Phys. Rev. Lett. 87142301 , (2001). X. N. Wang and X. F. Guo, Nucl. Phys. A 696, 788 (2001); X. F. Guo and X. N. Wang, Phys. Rev. Lett. $853591,(2000)$.

[10] N. Armesto, C. A. Salgado and U. A. Wiedemann, Phys. Rev. D 69, 114003 (2004).

[11] M. Djordjevic and M. Gyulassy, Phys. Lett. B 560, 37 (2003); and Nucl. Phys. A 733, 265 (2004).

[12] M. Djordjevic, Phys. Rev. C, Phys. Rev. C 73, 044912 (2006).

[13] Yu. Maezawa et al. [WHOT-QCD Collaboration], Phys. Rev. D 81091501 (2010); Yu. Maezawa et al. [WHOTQCD Collaboration], PoS Lattice 194 (2008).

[14] A. Nakamura, T. Saito and S. Sakai, Phys. Rev. D 69, 014506 (2004).

[15] A. Hart, M. Laine and O. Philipsen, Nucl. Phys. B 586, 443 (2000).

[16] D. Bak, A. Karch and L. G. Yaffe, JHEP 0708, 049 (2007).

[17] M. Djordjevic and U. Heinz, Phys. Rev. C 77, 024905 (2008).

[18] M. Djordjevic, M. Gyulassy, Phys. Rev. C 68, 034914 (2003).

[19] P. Aurenche, F. Gelis and H. Zaraket, JHEP 0205, 043 (2002).

[20] R.L. Kobes, G. Kunstatter, A. Rebhan, Phys. Rev. Lett. 64, 2992 (1990).

[21] E. Braaten, R.D. Pisarski, Phys. Rev. D 42, 2156 (1990).

[22] M. Dirks, A. Niegawa, K. Okano, Phys. Lett. B 461, 131 (1999).

[23] P. Arnold, G. D. Moore, L. G. Yaffe, JHEP 0111, 057 (2001); JHEP 0206, 030 (2002); JHEP 0301, 030 (2003).

[24] A. Rebhan, Lect. Notes Phys. 583, 161 (2002); 